\title{
ÉDITORIAL
}

\section{HISTOIRE DES ENTREPRISES SOCIALISTES : CHANTIERS EN COURS par Gilles GUIHEUX}

Professeur de socio-histoire

Université de Paris

Membre de l'Institut Universitaire de France

Centre d'études en sciences sociales sur les mondes africains, américains et asiatiques

Texte paru dans Entreprises et histoires, 2021/2 (nº 103), pages 6 à 12

Les économies socialistes ont d'emblée suscité l'intérêt des économistes, et ce d'abord au sein même du monde socialiste. On pense bien sûr à János Kornai qui, pendant plus de deux décennies, a été chercheur à l'Institut d'économie de l'Académie hongroise des sciences avant de rejoindre les Etats-Unis au milieu de la décennie 1980. Les historiens ont aussi mis en perspective l'expérience socialiste ; Iván T. Berend et György Ránki écrivent pour leur part une histoire comparative de l'industrialisation hongroise ${ }^{1}$. A l'Ouest, notamment du fait d'une demande émanant des diplomates et des militaires, en particulier aux Etats-Unis, économistes et géographes développent des travaux sur la base de statistiques publiées par les administrations nationales, de voyages d'études - lorsqu'ils sont possibles - ou de l'examen de sources locales, parfois traduites ${ }^{2}$. L'émigration permet le développement de travaux de sociologie et de science politique, et les débuts d'une histoire orale ${ }^{3}$; Andrew G. Walder prend par exemple appui sur des témoignages recueillis à Hong Kong pour écrire un ouvrage fondateur sur l'entreprise chinoise socialiste ${ }^{4}$. Dans les années 1980, les frontières s'ouvrent et des travaux de terrain sont désormais possibles ${ }^{5}$.

Avec la chute des régimes d'Europe de l'Est en 1989, les études historiques sur le communisme connaissent dans de nombreux pays un essor et un profond renouvellement lié à

\footnotetext{
${ }^{1}$ I. T. Berend et G. Ránki, The Hungarian Economy in the Twentieth Century, Londres, Croom Helm, New York, Saint Martin's Press, 1985.

${ }^{2}$ Les rapports du Joint Publications Research Service (JPRS) rendent accessibles en langue anglaise des documents originellement publiés par la presse écrite, les médias radiophoniques et la presse technique spécialisée. Créé en 1957 à l'initiative du Département du Commerce, le JPRS fonctionne jusqu'en 1995.

${ }^{3}$ En 1957-1958, l'Université Columbia conduit un projet d'histoire orale avec plusieurs centaines de réfugiés hongrois, le Columbia University Research Project on Hungary (CURPH). Voir : https://www.osaarchivum.org/digital-repository/osa:ea9bfcca-2dd5-4da8-b6a4-74871c5373f4.

${ }^{4}$ A. G. Walder, Communist Neo-Traditionalism: Work and Authority in Chinese Industry, Berkeley, University of California Press, 1986.

${ }^{5}$ Voir V. Nee et D. Stark (eds.), Remaking the Economic Institutions of Socialism, Stanford, Stanford University Press, 1989.
} 
l'ouverture des archives officielles des États et des partis communistes jusque-là en grande partie inaccessibles. Une approche diachronique de l'histoire des entreprises devient possible $^{6}$; nombre de ces travaux sont présentés à la Business History Conference ${ }^{7}$. En France, grâce à l'accès à ces sources nouvelles, Sandrine Kott réalise un des tout premiers ouvrages consacrés à l'histoire des entreprises socialistes d'après la Seconde Guerre mondiale ${ }^{8}$. Cependant Sandrine Kott ne s'intéressait pas à l'entreprise comme un lieu de production, mais en tant qu'organisation et institution centrale, prenant en charge la vie sociale, culturelle et politique de ses salariés. C'est une analyse politique de l'entreprise qu'elle conduit, considérant l'entreprise industrielle comme un lieu d'observation des interactions entre le pouvoir et les individus.

Ce n'est que plus récemment que l'histoire des entreprises a pris pour objet les activités économiques dans les pays socialistes ${ }^{9}$. Dans leur ouvrage programmatique paru en 2013, Philip Scranton et Patrick Fridenson appelaient à «réimaginer» la discipline en élargissant les champs de l'enquête hors des sentiers battus de la grande entreprise capitaliste $^{10}$. Les deux auteurs invitaient à ouvrir des chantiers inexplorés et examinaient les possibilités de questions nouvelles. Dans la première partie consacrée aux pièges à éviter, quelques pages invitaient à ne pas considérer les États-Unis (et l'Occident en général) comme la norme, et à s'éloigner d'une vision qui ferait de l'entreprise capitaliste européenne et américaine le modèle unique amené à prévaloir.

Philip Scranton joue un rôle majeur dans le développement de la recherche sur l'histoire de la gestion des entreprises socialistes. Enterprise \& Society, dont il est ancien rédacteur en chef, publie en 2018 un texte qu'il consacre aux entreprises socialistes d'Europe centrale et orientale $(1945-1970)^{11}$, ainsi que plusieurs autres contributions qui dialoguent

\footnotetext{
${ }^{6}$ On se reportera par exemple aux travaux cités par M. Christian et E. Droit à propos d'entreprises de la RDA et de la Pologne («Ecrire l'histoire du communisme : l'histoire sociale de la RDA et de la Pologne communiste en Allemagne, en Pologne et en France », Genèses, n 61, 2005, p. 118-133).

7 https://thebhc.org. Des communications ont été également été présentées à l'European Business History Association.

${ }^{8} \mathrm{~S}$. Kott, Le communisme au quotidien. Les entreprises d'État dans la société est-allemande, Paris, Belin, 2001.

${ }^{9}$ Voir par exemple le travail de Pál Germuska sur l'industrie millitaire (P. Germuska, « L'industrie de la défense hongroise. De la soviétisation à l'occidentalisation », Vingtième siècle. Revue d'histoire, $\mathrm{n}^{\circ}$ 109, 2011, p. 89100).

${ }^{10}$ P. Scranton et P. Fridenson, Reimagining Business History, Baltimore, John Hopkins University Press, 2013.

11 P. Scranton, «Managing Communist Enterprises: Poland, Hungary, and Czechoslovakia, 1945-1970», Enterprise \& Society, vol. 19, $\mathrm{n}^{\circ} 3,2018$, p. $492-537$.
} 
avec lui ${ }^{12}$. L'article est le fruit d'une première exploration des pratiques de gestion en Pologne, Hongrie et Tchécoslovaquie ; Scranton met en évidence les évolutions entre l'avant et l'après 1956 et l'originalité de ces entreprises relativement à leurs homologues capitalistes occidentales.

Plus récemment, en 2019, Philip Scranton consacre un ouvrage aux entreprises chinoises de la période 1949-1970 ${ }^{13}$. L'essentiel de la littérature jusque-là disponible soit porte sur les politiques macro-économiques, soit est circonscrite à des communautés le plus souvent rurales. L'intérêt de la démarche de Scranton est donc de se situer au niveau de l'entreprise ou de la branche industrielle. L'originalité de son propos tient à ce qu'il met au centre la question de l'expérimentation technique et organisationnelle, posée à l'échelle de l'entreprise dans une diversité de secteurs d'activité, de l'agriculture à la construction en passant par le commerce et l'industrie.

L'ouvrage fait entendre les voix des gestionnaires, des entrepreneurs, des cadres et des travailleurs de la Chine communiste, de 1949 jusqu'au début des années 1970. Comment les entreprises chinoises ont-elles fonctionné, évolué, expérimenté, improvisé ? Quelles initiatives technologiques ont été cruciales pour ces processus, nécessairement développés avec une expertise et des ressources financières limitées ? Comment la construction du socialisme a-t-elle pu contribuer à jeter les bases de la croissance de l'après-1980, la République Populaire de Chine entrant avec confiance dans le $\mathrm{XXI}^{\mathrm{e}}$ siècle alors que les socialismes soviétique et d'Europe centrale s'effondraient? Et que pourraient apprendre les managers et entrepreneurs occidentaux d'aujourd'hui des pratiques et performances chinoises d'il y a un demi-siècle? Telles sont les principales questions posées.

C'est l'accès à des sources nouvelles en langue anglaise qui a rendu possible ce travail. Il s'agit pour partie de documents autrefois inaccessibles de la CIA désormais déclassifiés ; celle-ci, soucieuse de mieux connaître les pays socialistes durant la guerre froide, employait des traducteurs à transcrire en langue anglaise ce que produisaient les médias, notamment radiophoniques, des pays du bloc socialiste. D'autres sources, notamment en provenance du Joint Publications Research Service, financé par le Département de la Défense, rassemblent des traductions de publications chinoises techniques et scientifiques qui sont notamment

\footnotetext{
${ }^{12}$ Parmi les contributeurs figure Pál Germuska («What Can We Learn from the Business History of Communist Enterprises?», Enterprise \& Society, vol. 19, $\mathrm{n}^{\circ}$ 3, 2018, p. 538-545) qui contribue au présent numéro d'Entreprises et Histoire.

${ }^{13}$ P. Scranton, Enterprise, Organisation and Technology in China. A Socialist Experiment, 1950-1971, Cham, Palgrave Macmillan, 2019. L'auteur a terminé pour le même éditeur la moitié d'un second livre, consacré aux entreprises socialistes en Hongrie de 1945 à 1970 ("from chaos to contradictions").
} 
organisées par secteurs industriels. Ces matériaux digitalisés sont désormais aisément consultables. S'intéresser au cas chinois paraît d'autant plus urgent que la République populaire est désormais la deuxième puissance économique mondiale, ce qui explique aussi la place qu'elle occupe dans ce numéro dirigé par un socio-historien de la Chine.

Les initiatives se multiplient pour donner accès aux sources. On citera ici deux initiatives qui visent à rassembler des matériaux sur lesquels élaborer de futurs travaux. Le premier est la base de données en cours de construction par Zhaojin Zeng, qui a déjà publié dans cette même revue ${ }^{14}$. Le Chinese Factory Project (CFP) est un projet d'histoire numérique qui vise à collecter, analyser et publier des sources archivistiques et quantitatives relatives à l'histoire des entreprises, au développement industriel et à la vie économique de la Chine moderne ${ }^{15}$. Financé par Duke Kunshan University, le CFP a initié trois chantiers : une collection d'archives numériques ; une base de données qui comprendra à terme plus de 6000 entreprises; des représentations visuelles des évolutions spatiales et écologiques de la transformation économique de la Chine au $\mathrm{XX}^{\mathrm{e}}$ siècle. Dans ce numéro, Zhaojin Zeng et ses co-auteurs commentent deux documents extraits de ces archives. Un second exemple est l'initiative d'Anna Pikos et Tomasz Olejniczak qui ont construit une base de données de 387 entreprises polonaises ayant plus d'un siècle d'histoire ${ }^{16}$.

\section{CHANTIERS EN COURS}

Ce numéro d'Entreprises et Histoire participe donc d'une riche actualité scientifique. L'historiographie la plus récente des entreprises socialistes pose de multiples questions. Se pose par exemple celle des chronologies qui ne sont pas nécessairement celles de l'histoire politique, comme le montre la contribution de Robert Cliver dans ce numéro à propos des tentatives de cogestion des entreprises du secteur de la soie à Shanghai au début des années 1950. Il est sans doute nécessaire de se défaire de l'exceptionnalisme historique attribué au communisme, qui pousse à analyser les phénomènes sociaux comme procédant uniquement de la nature politique des régimes.

\footnotetext{
${ }^{14}$ Z. Zeng, «Company archives and business history in contemporary China: the case of the Baojin company archive », Entreprises et Histoire, n ${ }^{\circ}$ 90, avril 2018, p. 145-148.

${ }^{15}$ Consultable à l'url : https://chinesefactory.org.

${ }^{16}$ A. Pikos et T. Olejniczak, «Business History in Poland: Current State and Future Potential », Journal of Management and Business Administration, Central Europe, vol. 25, n 3, 2017, p. 55-77.
} 
Ailleurs, dans un ouvrage qui vient de paraître ${ }^{17}$, Robert Cliver montre combien, en matière de gestion des populations ouvrières de Chine, les situations étaient contrastées au sein d'un même secteur d'activité. Basé sur des archives chinoises et centré sur les années 1950, l'ouvrage compare deux groupes de travailleurs de la soie et leur expérience de la révolution. Les tisserands de soie masculins des usines de Shanghai entretenaient des liens étroits avec le Parti Communiste et ont largement bénéficié des politiques socialistes après 1949. En revanche, les ouvriers des filatures étaient pour la plupart des jeunes femmes qui n'avaient ni organisations puissantes, ni liens avec le régime révolutionnaire. Pour nombre d'entre elles, les conditions de travail changèrent peu après 1949 et les campagnes politiques de mobilisation pour accroître la production aggravèrent le caractère brutal et oppressif du régime de travail caractéristique des usines depuis le XIX ${ }^{\mathrm{e}}$ siècle. À la fin des années 1950, les conflits et les privations se généralisèrent parmi les ouvriers de la soie et, malgré son impressionnante reprise sous le régime communiste, l'industrie fut confrontée à une crise pire que la guerre et la révolution. Le monde socialiste est donc hétérogène, d'un pays à l'autre, mais aussi au sein d'un même pays, ou d'une même région, ou d'un même secteur.

Dans ce numéro, deux autres contributions reviennent sur les modalités de la gestion des populations ouvrières. Emmanuel Droit, à partir d'archives inédites, évoque le cas d'une entreprise est-allemande de landaus dans les années 1970. Il montre que l'entreprise d'État, outre un lieu de production matérielle, était aussi un espace de construction des identités sociales, à la fois personnelles et professionnelles. En leur sein, le régime communiste a façonné pendant quarante ans des identités socio-professionnelles en valorisant la notion de travail. Ce système productif allait de pair avec une politique sociale très généreuse et un fonctionnement communautaire. Quatre décennies plus tard, dans les années 2010, en Chine, Judith Audin explore un ancien quartier industriel et ouvrier de la capitale. Une partie des installations industrielles sont patrimonialisées, tandis que les populations anciennement ouvrières, et leurs espaces d'habitation, disparaissent progressivement sous le coup de la promotion immobilière et de la gentrification.

Cette historiographie s'intéresse aussi aux rapports entre entreprises et État. La revue Business History a ainsi mis en ligne en 2020 les deux premiers articles d'un numéro spécial à paraître sur «Socialist entrepreneurs: Business histories of the GDR and Yugoslavia » qui est codirigé par Vladimir Unkovski-Korica et Sasa Vejzagic. Pieter Troch analyse l'histoire

\footnotetext{
${ }^{17}$ R. Cliver, Red Silk. Class, Gender, and Revolution in China's Yangzi Delta Silk Industry, Cambridge, MA, Harvard University Asia Center, 2020.
} 
d'une entreprise de taille moyenne de transformation du bois dans la Yougoslavie socialiste des années $1960^{18}$. Il explore les conditions de la réorganisation de l'entreprise conduite dans le cadre des réformes économiques orientées vers le marché, et s'intéresse en particulier aux rapports entre gestionnaires, autorités locales et autorités centrales. De son côté, Max Trecker étudie la relation ambiguë entre l'État-Parti et l'entrepreneuriat privé en République démocratique allemande $^{19}$. Il se centre sur la fin des années 1980 et sur le rôle que les entrepreneurs privés étaient censés jouer dans la réforme et le sauvetage du socialisme en Allemagne de 1'Est.

Plusieurs autres questions sont actuellement étudiées. Il en va ainsi des capacités de certaines entreprises socialistes nationalisées à préserver leur identité face aux pressions des pouvoirs politiques dans le sens de la conformité à leurs desiderata, comme l'a montré l'étude sur longue période d'une entreprise est-allemande spécialisée dans la production de meubles et d'éléments d'intérieur par une historienne et un professeur de gestion ${ }^{20}$. D'autres auteurs abordent les complexes processus de prise de décision au sein des organes gouvernementaux entre fonctionnaires techniciens et militants politiques. D'autres encore interrogent le compartimentage entre des ministères économiques cloisonnés, parfois en discordance avec les réalités (l'exemple en sens contraire étant le puissant ministère des Chemins de fer de la Chine $)^{21}$.

Pour les entreprises socialistes de tous les pays apparaissent cependant les coups de grisou que peuvent représenter les brusques réorientations politiques. Ainsi, en Chine, l'équipement des rails victime de la «bataille de l'acier » durant le Grand bond en avant ou les billets de train gratuits accordés durant la Révolution culturelle aux Gardes rouges pour qu'ils portent la révolution dans tout le pays ${ }^{22}$.

Cette question des rapports entreprises-État est travaillée par plusieurs des contributions à ce numéro, notamment à propos des entreprises automobiles du Comecon et surtout dans le cadre des mutations des entreprises d'État lors de la sortie de l'économie

\footnotetext{
${ }^{18} \mathrm{P}$. Troch, «Tensions between plan and market in a political factory in socialist Kosovo », Business History, online version, March 2020, https://doi.org/10.1080/00076791.2020.1733981.

${ }^{19}$ M. Trecker, «Entrepreneurs as saviours of socialism? The complicated relationship between East German state socialism and entrepreneurship », Business History, March 2020, https://doi.org/10.1080/00076791.2020.1781818.

${ }^{20}$ K. Schreiter and D. Ravasi, «Institutional Pressures and Organizational Identity: The Case of Deutsche Werkstätten Hellerau in the GDR and Beyond, 1945-1996 », Business History Review, vol. 92, n 3, 2018, p. 453-498.

${ }^{21}$ E. Köll, Railroads and the Transformation of China, Cambridge, MA, Harvard University Press, 2019, IV partie.

${ }^{22}$ Ibid.
} 
planifiée ${ }^{23}$. Dans le cas vietnamien, Khac Minh Tran étudie une société locale de commerce de produits agricoles et de consommation née en 1976, transformée en 1986 en compagnie d'import-export et devenue un conglomérat diversifié, fortement investi dans la promotion immobilière grâce à son ancrage territorial et aux soutiens des autorités provinciales. Dans le cas cubain, dans une perspective néo-institutionnaliste, Seida Barrera Rodriguez et Ernesto Dominguez Lopez analysent les transformations du cadre réglementaire propre aux entreprises d'État.

Dans ce numéro, la question de l'innovation est abordée par trois contributions ${ }^{24}$. Alexandra Koulaeva revient sur la figure d'Alexeï Gastev (1882-1939), théoricien soviétique de l'organisation du travail à l'esprit utopique et imaginatif. Gastev fonde à Moscou un Institut central du Travail qui formera des centaines d'ingénieurs appelés à moderniser l'industrie soviétique. Valentina Fava compare, quant à elle, deux entreprises automobiles, la tchécoslovaque Škoda (à Mladá Boleslav) et la soviétique VAZ (Voljskiy Avtomobilny Zavod, à Togliatti) du point de vue des conditions de l'innovation technologique et de produit durant la période 1960-1980. Elle montre combien les trajectoires distinctes des deux entreprises s'expliquent par des accès différentiels aux technologies étrangères et par des usages différents. À l'échelle de la Pologne, Philip Scranton choisit d'explorer l'entretien et les réparations, une dimension du cycle de vie des produits et des infrastructures longtemps négligée par les chercheurs ${ }^{25}$. Alors que le système socialiste donne la priorité à la reconstruction de l'appareil industriel puis à l'augmentation de la production, ce secteur est très largement négligé et sous-financé, ce qui contribuera à l'écroulement du socialisme.

La question de l'internationalisation des entreprises est abordée par Julien Vercueil à propos de deux entreprises de l'énergie emblématiques de l'économie russe contemporaine, Rosneft et Gazprom, qui suivent des voies différenciées associées à des distances différentes à

\footnotetext{
${ }^{23}$ Sur le cas chinois, on pourra se reporter à G. Guiheux, «Le renouveau du capitalisme familial. Défense et illustration par un entrepreneur du Zhejiang », Perspectives chinoises, $\mathrm{n}^{\circ}$ 87, janvier-février 2005, p. 22-32 et G. Guiheux, «D'une entreprise d'État à un marché spécialisé. La reconversion d'un espace économique urbain dans la province du Hunan », Perspectives chinoises, ${ }^{\circ} 78$, juillet-août 2003, p. 4-17.

${ }^{24}$ Sur l'innovation, voir B. Kogut et U. Zander, « Did Socialism Fail to Innovate? A Natural Experiment of the Two Zeiss Companies », American Sociological Review, vol. 65, n 2, 2000, p. 169-190. Les auteurs comparent deux sociétés, l'une en Allemagne de l'Ouest, l'autre en Allemagne de l'Est, séparées à l'issue de la Seconde Guerre mondiale.

${ }^{25}$ K. L. Borg, « Les sens perdus du garagiste. Comment le savoir-faire a été disqualifié dans l'univers automobile américain », Revue d'histoire moderne et contemporaine, vol. 59, $\mathrm{n}^{\circ}$ 3, 2012, p. 19-47 ; A. L. Russell and L. Vinsel, «After Innovation, Turn to Maintenance », Technology and Culture, vol. 59, $\mathrm{n}^{\circ}$ 1, 2018, p. 1-25 ; S. Krebs, G. Schabacher und H. Weber (Hg.), Kulturen des Reparierens. Dinge - Wissen - Praktiken, Bielefeld, transcript, 2018 ; S. Krebs and H. Weber (eds.), Histories of Technology's Persistence: Repair, Reuse and Disposal, Bielefeld, transcript, à paraître.
} 
l'État russe. D'autres travaux explorent les formes d'internationalisation, de coopération et de domination au temps du socialisme, notamment à l'échelle du Conseil d'assistance économique mutuelle (CAEM, plus connu sous son acronyme anglais Comecon), disparu le 28 juin 1991, et dont le trentième anniversaire de la désintégration est commémoré cette année $^{26}$. Simon Godard vient de publier une histoire sociale de cette institution qui montre que le CAEM a joué un «rôle de laboratoire d'acculturation transnationale » pour ses fonctionnaires avant de «devenir, paradoxalement, un espace de contestation de l'hégémonie soviétique $»^{27}$. Dans sa contribution, Pál Germuska commente les collections relatives au CAEM au sein des Archives nationales de Hongrie et souligne combien la variété des archives désormais disponibles autorise des recherches neuves. En revanche, le choix a été fait de ne pas traiter dans ce numéro du contrôle des échanges avec les entreprises socialistes menés par les pays capitalistes après 1949 ; il a eu des conséquences profondes, a évolué au cours du temps et a suscité des tensions internes. Son étude a été renouvelée par l'ouverture progressive des archives à l'Ouest ${ }^{28}$.

Un autre champ actif de recherches est celui des efforts conduits par les pays capitalistes pour tirer partir du potentiel des marchés et entreprises socialistes. Des travaux ont été récemment publiés sur les relations bancaires franco-polonaises ${ }^{29}$ et sur les relations économiques franco-chinoises jusqu'en $1964^{30}$, ou entre firmes, tel un transfert de technologie

\footnotetext{
${ }^{26}$ Voir le programme de la conférence organisée pour les 30 ans de la désintégration du CAEM organisée par le Centre de Recherche Europes-Eurasie (CREE) de l'Inalco: http://www.inalco.fr/evenement/conferenceinternationale-trente-ans-apres-desintegration-caem-enseignements-europe.

${ }^{27}$ S. Godard, Le laboratoire de l'internationalisme. Le CAEM et la construction du bloc socialiste (1949-1991), Paris, Presses de Sciences Po, 2021.

${ }^{28}$ Pour une sélection partielle de références : «Multilateral Export Control Policy: The Coordinating Committee (CoCom) », in R. Goldberg (ed.), Technology and East-West Trade, Washington, Government Printing Office, 1979, p. 153-172 ; G. K. Bertsch (ed.), Controlling East-West Trade and Technology Transfer. Power, Politics, and Policies, Durham, Duke University Press, 1988 ; Y. Yasuhara, «The myth of free trade: the origins of COCOM 1945-1950», The Japanese Journal of American Studies, vol. 4, 1991, p. 127-148 ; M. Mastanduno, Economic containment: CoCom and the politics of East-West trade, Ithaca, Cornell University Press, 1992 ; E. H. Noehrenberg, Multilateral export controls and international regime theory: the effectiveness of COCOM, Sinzheim, Pro Universitate Verlag, 1995 ; L. Segreto, «Europe vs. United States or bellum omnium contra omnes? COCOM, national economic interests, pressure groups and politics in East-West trade in the 1950's and 1960's ", in R. P. Amdam, A. E. Hagberg and K. Sogner (eds.), Proceedings from the EBHA Conference, 31 st August - 1st September 2001, Oslo, University of Oslo, 2002 ; I. R. W. Jackson, « Waging the Economic Cold War: Britain and CoCom, 1948-54», in M. F. Hopkins, M. D. Kandiah, G. Staerck (eds.), Cold War Britain, 1945-1964, Londres, Palgrave, 2003, p. 41-54 ; L. Segreto, «East-West Trade in Cold War Europe: National Interests and Hypocrisy », in A. Tonini (ed.), Towards a New Europe: Identities, Economics, and Institutions, Florence, Polistampa, 2006.

${ }^{29}$ E. Mourlon-Druol, «Banking on détente: Barclays, Paribas, and Société Générale in Poland, 1950s1980s », Business History, online version, 2021 , https://www.tandfonline.com/doi/abs/10.1080/00076791.2021.1907347?journalCode=fbsh20.

${ }^{30}$ T. Robin, Le Coq face au Dragon. Deux décennies de relations économiques franco-chinoises de la fin de la Seconde Guerre mondiale au milieu des années 1960, Genève, Droz, 2013.
} 
d'une entreprise de RDA - la même que celle étudiée quinze ans plus tôt par les sociologues Bruce Kogut et Udo Zander - au profit d'une entreprise du Japon, facilitée par un accord de rapprochement économique entre les deux États ${ }^{31}$.

Les contributions à ce numéro mettent en évidence un ensemble de caractéristiques partagées de l'Europe à l'Asie socialiste, en passant par le cas cubain. Au sein d'une même économie, les entreprises sont de statuts divers ; elles relèvent tantôt de l'État central, tantôt des gouvernements locaux, tandis que se maintient, sous des formes variables, un secteur privé $^{32}$. Derrière la planification se cachent de multiples problèmes de coordination. Les contraintes en matière de capitaux, de personnel qualifié, d'accès aux technologies, expliquent une grande part les choix successifs des entreprises. Ce sont en outre des pratiques en marge des normes, ou carrément illicites, qui peuvent permettre aux entreprises et aux ménages de perdurer face aux pénuries et aux rigidités. Symétriquement, face à l'autorité verticale des administrations et du Parti, il existe un pouvoir muet des salariés, ouvriers ou personnels d'encadrement, fondé sur des connaissances tacites et la pratique des lieux et des objets, qui peut leur donner de l'influence, ici comme ailleurs ${ }^{33}$.

Le numéro s'achève par un débat entre trois universitaires (deux économistes, une enseignante-chercheuse en gestion) et trois praticiens (un industriel, un banquier, un consultant) sur les conditions de passage, vues depuis l'intérieur des entreprises, de systèmes d'économies planifiées à des formes d'économie de marché. La question des héritages, pour le secteur public comme pour le secteur privé, et des nouvelles normes comptables ou de gestion est posée à l'épreuve d'une variété de cas nationaux, dont celui de la Corée du Nord ${ }^{34}$. Il en ressort une grande variété de trajectoires, dont la plus brillante et sans doute la plus inattendue est celle de la Chine ${ }^{35}$.

\footnotetext{
31 A. Kudo, «Economic relations between Japan and East Germany: the developing relationship of Kureha Kagaku and Carl Zeiss Jena after the first oil crisis », Entreprises et Histoire, n' ${ }^{80}$, septembre 2015, p. 84-113.

32 Adam K. Frost, dans une thèse en cours à Harvard et intitulée "'Speculators and Profiteers': The Entrepreneurial Transformation of Socialist China (1957-1978) », soutient que dans les années 1970 l'entreprise privée est si répandue que l'État chinois n'a plus les moyens de limiter son développement. Ce serait donc les forces spontanées du capitalisme qui auraient conduit aux réformes et non, comme le régime le répète à l'envi, la volonté politique de Deng Xiaoping.

${ }^{33}$ R. C. H. Chia and R. Holt, Strategy Without Design. The Silent Efficacy of Indirect Action, Cambridge, Cambridge University Press, 2009.

${ }^{34}$ P. Pons, Corée du Nord. Un État-guérilla en mutation, Paris, Gallimard, 2015 ; J. V. Hastings, A Most Enterprising Country: North Korea in the Global Economy, Ithaca, Cornell University Press, 2016.

${ }^{35} \mathrm{Ce}$ numéro n'aurait pas été possible sans la collaboration active de Hadrien Coutant, Patrick Fridenson et Philippe Lefebvre auxquels l'auteur adresse ses plus vifs remerciements. L'idée d'origine en revient à Frédéric Garcias (sciences de gestion, Université de Lille), alors secrétaire de rédaction de la revue, qui l'a suggérée au comité de rédaction en mai 2016.
} 